\title{
RELAÇÕES PÚBLICAS NA CHINA: o desafio do guanxi
}

\author{
Public Relations in China: the guanxi's challenge
}

\author{
Aline Bastos ${ }^{\mathrm{a}}$, Aguirre Estorilio Silva Pinto Neto ${ }^{\mathrm{b}}$ \\ a Relações Públicas, Jornalista, Mestranda em Comunicação e Cultura pela ECO-UFRJ. Trabalhou na área de Comunicação do \\ Instituto Brasil-China, onde começou a pesquisar sobre a China e as relações sino-brasileiras. Rio de Janeiro - RJ, Brasil, e-mail: \\ aline.bastos@gmail.com. \\ b Relações Públicas, Especialista em Comunicação Empresarial pela Universidade de Gestão e Negócios da Petrobras. Ex-diretor \\ de comunicação do Instituto Brasil-China, morou por mais de três anos na China. Rio de Janeiro - RJ, Brasil, e-mail: \\ aguirre.neto@gmail.com.
}

\begin{abstract}
Resumo
Este trabalho apresenta as diferentes linhas de pesquisa de Relações Públicas Internacionais, destacando as teorias culturais de Wakefield (1996) para o estudo e para a elaboração de políticas e estratégias de Relações Públicas em outros países, sobretudo na China. Destaca aspectos da tradição cultural milenar chinesa e sua contribuição para a ascensão da China no cenário internacional. Descreve as formas de introdução e de consolidação da prática de Relações Públicas na China, ressaltando uma característica cultural peculiar do povo chinês - o guanxi, uma rede de relacionamentos pessoais indispensáveis ao funcionamento social e político do país - que desafia a prática de Relações Públicas, segundo o modelo americano.
\end{abstract}

Palavras-chave: Relações Públicas internacionais; Aspectos culturais chineses; Relações Públicas na China; Guanxi.

\begin{abstract}
This article presents the different lines of research of International Public Relations, presenting the cultural theories of Wakefield (1996) for the study and the elaboration of Public Relations politics and strategies in other countries, specially in China. It shows aspects of the millenary Chinese cultural tradition and its contribution for the ascension of China in the international scene. It describes the forms of introduction and consolidation of the practice of Public Relations in China, standing out a peculiar cultural characteristic of the Chinese people - the guanxi, a net of personal relationships indispensable to the social and political functioning of the country that challenges the practice of Public Relations, according to the American model.
\end{abstract}

Keywords: International Public Relations; Chinese cultural aspects; Public relations in China; Guanxi.

Rev. Estud. Comun., Curitiba, v. 9, n. 19, p. 113-122, maio/ago. 2008 


\section{INTRODUÇÃO}

A civilização chinesa, a mais antiga do mundo, sempre desperta a atenção mundial pelos seus mais de cinco mil anos de história, pela população de mais de 1,3 bilhões de pessoas e pelo seu regime oficialmente comunista. Nos últimos anos, porém, há um interesse cada vez maior pelo país que mais cresce no mundo, e que caminha para se tornar uma superpotência mundial.

O Governo continua nas mãos do partido comunista chinês, longe do modelo de democracia ocidental; porém a abertura econômica nos moldes do capitalismo transformou a experiência chinesa em única no mundo e vem provocando profundas transformações mundiais. Tu Wei Ming (2002, p. 359) admite: "Essa identificação positiva [da China] com o Ocidente e a participação ativa na reestruturação fundamental do próprio mundo segundo o modelo ocidental não tem precedente na história humana." Do mesmo modo, afirma o famoso sociólogo espanhol Castells (1999, p. 348): "O fato do Império do Meio, rompendo com um padrão milenar de isolamento, ter se incorporado deliberadamente ao resto do mundo mudou o curso da história universal."

O estrondoso crescimento econômico e o poder político internacional proveniente desse fato colocam a China num patamar sócio-cultural privilegiado. "Há dez anos, ninguém prestava atenção na existência da China. Era um país a mais no globo. Hoje, todos querem saber o que está acontecendo aqui, como estamos vivendo, em que direção vamos, como pretendemos nos relacionar com o resto do mundo", diz o pesquisador Jiang Shixue $^{1}$ da Academia Chinesa de Ciências Sociais.

A investigação das Relações Públicas na China revela nuances culturais peculiares que podem contribuir para o sucesso das práticas de comunicação de instituições e organizações multinacionais que atuam ou pretendem atuar nesse país, bem como para ampliar as formas de atuação do profissional em qualquer outro país do mundo. Compreender a riquíssima tradição cultural de um país com mais de cinco mil anos de história, e que desponta como a nova superpotência do milênio, revela-nos que existem outros modos de ser, de agir e de pensar, que interferem decisivamente na aplicação das políticas e estratégicas de Relações Públicas pelo mundo.

Dessa forma, esse trabalho busca provocar a reflexão e o pensamento crítico sobre a atuação do profissional de Relações Públicas em esferas internacionais, ressaltando a importância do conhecimento e do respeito às diferenças culturais para a excelência das práticas comunicacionais em diferentes países.

\section{Relações Públicas internacionais}

Heath (2001, p. 625) descreve o que ele chama de "ironia da era da globalização" como "um sentimento de que o globo está simultaneamente encolhendo a medida em que se expande em um caleidoscópio crescente de povos, línguas, culturas, estruturas governamentais e sistemas econômicos." Essa é apenas uma das contradições desse fenômeno mundial de integração de mercados e economias, queda das barreiras alfandegárias, homogeneização de padrões culturais e alteração da ordem global.

Dentro desse contexto, como uma organização que fala para públicos de diferentes países (e culturas) pode corresponder a expectativas diversas sem perder sua identidade no processo? Essa questão é central para o estudo e a prática de relações públicas internacionais, uma vez que:

Relações Públicas Internacionais podem ser definidas como o esforço planejado e organizado de uma empresa, instituição ou governo em estabelecer relações mutuamente benéficas com os públicos de outras nações. Estes públicos, porsua vez, podem ser definidos como os vários grupos que são afetados, ou podem afetar as operações de uma firma, instituição ou governo em particular. (WILCOX et al., 2003, p. 378).

Os estudos sobre as relações públicas internacionais ainda são recentes, mas a velocidade com que pequenas e grandes organizações estão se internacionalizando está promovendo um interesse e um debate cada vez maior sobre o assunto. Com as novas tecnologias e a globalização que ocorre nos negócios, mais e mais os profissionais de relações

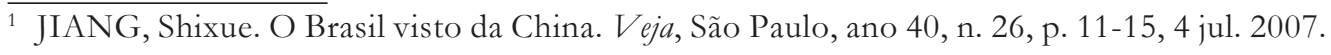

Rev. Estud. Comun., Curitiba, v. 9, n. 19, p. 113-122, maio/ago. 2008 
públicas necessitarão ser hábeis para se comunicarem de forma ampla com audiências internacionais.

As pesquisas na área de relações públicas internacionais tem seguido quatro tendências. A primeira linha "estende a idéia de relações públicas como uma atividade de comunicação simétrica proposta pelo modelo de Grunig" (TAYLOR, 2001). A segunda linha descreve e contextualiza a prática de relações públicas em diferentes países. A terceira linha tem o seu foco nos fundamentos pedagógicos e éticos das relações públicas internacionais, e uma quarta linha refere-se a pressuposições teóricas sobre o papel das relações públicas em cada nação.

Quando combinadas, observa Taylor (2001, p. 637), "as quatro linhas oferecem um retrato amplo do que nós sabemos e não sabemos sobre a prática (de relações públicas internacionais)". Nesse sentido, é importante considerar que o estudo das relações públicas internacionais acaba contribuindo para o conhecimento geral das relações públicas, ampliando as possibilidades de atuação dos profissionais da área, até mesmo em seus próprios países.

A virada internacional da prática de relações públicas beneficia o corpo acadêmico e os profissionais do mercado, pois "refletindo sobre nossas próprias normas culturais e sociais, nós nos tornamos mais sensíveis, críticos e abertos a novas perspectivas" (TAYLOR, 2001, p. 637).

O planejamento e o desenvolvimento de programas entre os países levam em conta aspectos culturais, econômicos, tradição, religião, entre outros, que adaptam os planos, estratégias e políticas de Relações Públicas de companhias nacionais às realidades locais.

A prática de relações públicas internacionais pode ser entendida de acordo com Wakefield (1996), a partir de um modelo composto por quatro teorias: teoria da sociedade global, teorias culturais, teorias de administração comparativa e teorias de comunicação.

A teoria da sociedade global retrata os efeitos de uma globalização acelerada. Se uma sociedade é afetada por grandes mudanças, ocorre inicialmente uma divergência e negação às novas mudanças, até a sociedade adaptar-se a tais mudanças e revertê-las em convergências de idéias. Unidades de relações públicas, se bem treinadas em análise ambiental e comunicação estratégica, podem diminuir ou até mesmo acabar com turbulências dessas grandes mudanças em escala global.
Nas teorias culturais, Adler (1983, apud WAKEFIELD, 1996) identificou três determinantes de cultura: "é compartilhada por todos os membros de algum grupo; membros mais velhos do grupo transmitem-na para os membros mais jovens; e por meio da moral, leis e costumes, a cultura molda o comportamento e as visões de mundo do grupo." Assim, a cultura exerce grande influência nos trabalhos de relações públicas internacionais. É fundamental que esse profissional conheça os aspectos culturais que culminam no ambiente no qual está inserido e que interfiram na elaboração de políticas e estratégias comunicacionais.

As teorias de administração comparativa também podem auxiliar a pesquisa internacional. De acordo com Adler (1983 apud WAKEFIELD, 1996), o domínio da administração comparativa expande o conhecimento doméstico comparando as decisões gerenciais e os comportamentos de uma cultura com aquelas de outra cultura. Busca por diferenças e similaridades entre a prática em um ou mais países visando formular princípios gerais de aplicação.

Teorias de gerenciamento comparativo frequentemente apóiam teorias de contingência. Katz e Kahn (1978 apud WAKEFIELD, 1996) determinaram que organizações efetivas mantêm uma perspectiva de sistemas abertos, significando que o ambiente externo é tão importante para elas quanto seus processos internos. Elas sabem que o ambiente pode mudar rapidamente e, por isso, criam programas para capacitar adaptações a mudanças.

Brinkerhoff e Ingle argumentaram que nem a abordagem da cultura específica e nem a da livre cultura é efetiva sozinha (WAKEFIELD, 1996). Eles mediam os pólos dizendo que organizações combinam valores de cultura específica (cultura adotada pela organização) e de livre cultura (diferentes culturas organizacionais). A teoria de Brinkerhoff-Ingle oferece esperança para relações públicas internacionais, porque profissionais também enfrentam decisões sobre o que centralizar e o que conduzir localmente.

Finalmente, as teorias de comunicação sugerem três implicações teóricas para relações públicas internacionais. As implicações envolvem a natureza das mensagens de mídia, o imperialismo da mídia e seus impactos e a proliferação de questões globais e ativismo.

Para Grunig (1994), os efeitos dos meios podem interessar aos profissionais de Relações Públicas internacionais porque as imagens que eles 
formam criam problemas às empresas e instituições multinacionais. As imagens ajudam a fomentar hostilidades sobre as organizações e estas necessitam desses profissionais para entenderem os atores essenciais desses ressentimentos e interferirem nos grupos afetados, antes do surgimento de insatisfações.

Profissionais de relações públicas internacionais devem estar qualificados e sensíveis a pontos de vista diversos, de modo a facilitar os relacionamentos entre diferentes públicos numa arena global. Além disso, podem também antecipar os desafios ajudando as organizações a pensar e a agir de maneira estratégica.

Para isso, principalmente, deve-se conhecer e respeitar as diferenças culturais do local das ações comunicacionais, como prevê a linha das teorias culturais de Wakefield (1996), bem como as diversas formas de hibridização e transculturação contemporânea. Essa percepção é fundamental para organizações e instituições multinacionais que atuam com públicos de diferentes países e culturas.

\section{A ascensão da China: a contribuição da tradição cultural}

A China desponta como a próxima superpotência mundial do século XXI. A experiência chinesa adotou o desempenho promovido pelo desenvolvimento econômico em ascensão e a legitimidade nacionalista, proporcionada pela invocação das características próprias de sua cultura. O líder socialista, Deng Xiaoping, construiu uma ampla reforma e implantou a diretriz de construir uma economia nacional forte, baseada no programa das quatro grandes modernizações: agricultura, indústria, ciência técnica e defesa nacional. Nas últimas décadas, o êxito dessas políticas se concretizou num concentrado e extraordinário ciclo de crescimento econômico e industrial a uma taxa média anual de $10 \%$, levando a China a ocupar posição junto aos líderes da economia mundial. Segundo estudos de bancos de investimentos mundiais publicados amplamente pela mídia, a China se tornará a maior potência econômica mundial nas próximas décadas por volta do ano 2030, mas possivelmente antes disso se continuar no atual ritmo de crescimento. Entretanto, para os chineses o mais importante são a harmonia e a prosperidade nacional com a evolução da sociedade chinesa, para se constituir a "sociedade harmônica", atual slogan do país.
O forte planejamento da China nas últimas décadas baseia-se na visão de mundo dos antigos filósofos chineses, sobretudo Confúcio, segundo os quais todos devem viver em relações harmoniosas e ter padrões de vida parecidos. "A ideologia política confuciana foi eficiente no desenvolvimento do Japão e dos quatro minidragões. Ela é evidente também nos processos políticos da República Popular da China, da Coréia do Norte e do Vietnã" (TU WEI MING, 2002, p. 360).

A cultura e a sociedade chinesas foram e continuam sendo influenciadas por Confúcio, filósofo chinês que viveu 500 anos antes de Cristo. O pensamento confuciano se constitui de três princípios fundamentais para a construção de uma sociedade ideal: Li, ou Estrutura; Ren ou Gentileza; e Xiao, ou Lealdade.

Lirefere-se à preservação de uma estrutura social baseada em uma ordem hierárquica, ou seja, as relações pessoais estão sempre em um par: governador e governado; pai e filho; irmão mais velho e irmão mais novo; marido e mulher; amigo e amigo. Cada um tem um papel bem marcado dentro do par hierárquico, tendo que cumprir certos deveres e assumir responsabilidades.

Ren refere-se ao amor, bondade e gentileza, elementos sem os quais não haveria harmonia da estrutura. Para Confúcio, a aquisição do Ren supõe vencer a si mesmo e a abertura de compreensão, solidariedade, amor, compaixão e compromisso com o outro. Assim, o Ren diz respeito a como deve ser a nossa postura frente ao tudo que pode haver entre duas pessoas - como se respeitar; como se amar; como se relacionar; como se entender; como lidar com as semelhanças e as diferenças, com a desarmonia, discordância e outros sentimentos humanos.

Em terceiro lugar vem Xiao, que é peça chave para a composição final da sociedade de Confúcio, e, refere-se à lealdade e obediência do subordinado a seu superior. Um cidadão deve ser leal ao seu governante, um filho deve obedecer a seu pai. Assim, enquanto $L i$ dita as estrutura das relações, Ren e Xiao permitem que essa estrutura se dê com harmonia.

A estrutura social hierarquizada, proposta pelo filósofo chinês, de papéis sociais bem definidos, contribuiu para o surgimento do guanxi, expressão que designa a complexa rede de relações pessoais indispensáveis ao funcionamento social e político 
na China. O guanxi - que possui o sentido de reciprocidade e confiança - é fortalecido com interações sociais, como visitas, presentes e convites para ocasiões especiais.

No diagrama de caleidoscópio proposto por Sandra Chen ${ }^{2}$ ilustram-se, além do guanxi, outros dois conceitos fundamentais à cultura chinesa. Cada elemento seria como uma face espelhada que refletiria infinitamente variadas imagens, à medida que se põe o caleidoscópio em movimento.

\section{GUANXI}

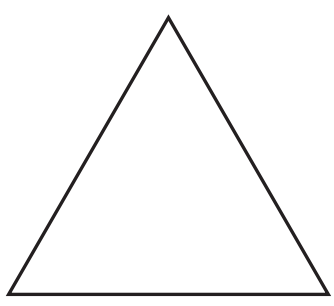

MIANZI

RENQING

FIGURA 1 - Localização da área de estudo

Aqui, o guanxi é apoiado pelos conceitos de renqing e mianzi.

Renqing indica generosidade, respeito ao próximo e sensibilidade, tudo isso em conformidade ao conceito de Confúcio sobre as obrigações de cada ator social em relação aos outros. A reciprocidade das relações na China pressupõe uma grande dose de gentilezas, expressa em formalidades e rituais de convivência. São regras implícitas do jogo social.

Mianzi, ou face, revela a preocupação dos chineses em preservar a reputação ou a honra. Perdese miañi ('cair a cara'), quando se viola o guanxi. É uma grande humilhação num contexto relacional.

Espera-se que as crianças preservem a "face" da família, ou o seu respeito social. Isto tem significado algo diferente em cada época. Para as crianças urbanas de hoje, significa ser bem educada e bem vestida, ganhar dinheiro e praticar valores tradicionais, como lealdade e bondade. Para os chineses das áreas rurais, significa colocar as necessidades do grupo na frente das necessidades individuais. Uma pessoa admirada é aquela que traz a maior honra para a família, enquanto continua sendo a mais humilde sobre conquistas pessoais. ${ }^{3}$ (CULTURE GRAMS, 2003, Não paginado).

De fato, toda essa filosofia humanista confuciana baseada na harmonia, gentileza, disciplina, família, responsabilidade, trabalho duro, coletivismo e abstinência remodelou os valores ocidentais que chegaram à China a partir da metade do século XX, e sugerem uma nova visão de mundo:

Certamente os valores iluministas, como a racionalidade instrumental, a liberdade, a consciência dos direitos, o devido processo legal, a privacidade e o individualismo são todos valores modernos universalizáveis, mas como sugere o exemplo confuciano, "valores asiáticos" como simpatia, justiça distributiva, consciência do dever, ritual, espírito público e orientação grupal também são valores modernos universalizáveis. Assim, os últimos podem se revelar uma referência crítica e oportuna para o estilo americano de vida. (TU WEI MING, 2002, p. 360).

\section{GUANXI: o desafio das Relações Públicas na China}

A morte de Mao Zedong, o fim da Revolução Cultural e a nova liderança de Deng Xiaoping inauguraram uma nova fase na história recente da China. Deng promoveu a abertura das portas da China ao ocidente na década de 80 e estabeleceu metas de modernização do país. A China abria-se irreversivelmente para conceitos ocidentais, entre eles, o de relações públicas. $\mathrm{Ni}$ Chen (1996, p. 122) afirma que: "o novo campo ajudava a facilitar transações, manter relações harmônicas e estabelecer relações mutuamente benéficas entre organizações e seus vários públicos em uma sociedade passando por várias mudanças."

É seguro dizer que na maior parte das vezes os primeiros contatos dos chineses com relações públicas deram-se pelas joint ventures

\footnotetext{
2 Sandra Chen é consultora de negócios entre a China e o Ocidente e ministrou oficina no seminário "China: como ter sucesso nos negócios", promovido pela Berlitz e a Câmara de Comércio e Indústria Brasil China, em março de 2004.

3 CULTURE GRAMS. Concise, reliable up-to date country reports on 200 cultures of the world. Newcastle: Axion Press, 2003. Disponível em: <www.culturegrams.com>. Acesso em: 15 jun. 2008. (Não paginado).
} 
ocidentais. Men (1990 apud NI CHEN, 1996) elaborou um breve roteiro das fases iniciais de implementação pelas quais as relações públicas passaram na China. Entre 1980 e 1985, o despertar de uma consciência no campo das relações públicas acompanhou os avanços da China em direção a uma economia de mercado. "Nesse estágio, desentendimentos surgiam, em parte, de preconceitos tradicionais. Profissionais confusos e desqualificados faziam má utilização das práticas existentes" (NI CHEN, 1996, p. 123).

A esta fase inicial seguiu-se uma explosão do interesse pelas relações públicas, que se seguiu até junho de 1989. Floresceram artigos e livros sobre o assunto, bem como associações de relações públicas e departamentos especializados. Também, nessa fase, verificou-se uma confusão a respeito dos objetivos e funções das relações públicas.

Mais e mais pessoas aprenderam a base e começaram a tentar utilizá-las [relações públicas]. Os princípios lentamente tornaramse melhor compreendidos. E, à medida que isso acontecia, o uso de relações públicas também se expandiu para novas áreas. (NI CHEN, 1996, p. 123).

O episódio ocorrido em 4 de junho de 1989, quando o governo reprimiu violentamente as manifestações estudantis pró-democráticas, deu origem a uma nova fase, em que a liderança do partido comunista resolveu rever todas as idéias importadas dos outros países.

Uma nova política enfatizou a definição de relações públicas socialistas com características chinesas. A política destacou que as relações públicas ocidentais não poderiam ser copiadas sem mudanças. Relações públicas com características chinesas, então, devem se desenvolver em concordância com características chinesas únicas. A China é um país socialista, governada por um governo comunista. Como consequência, tanto o partido quanto o governo devem supervisionar a prática no campo. (NI CHEN, 1996, p. 123).

Três anos depois, Deng Xiaoping fez uma visita histórica a várias cidades do sul da China e deixou claro em seus discursos que o processo de abertura da nação continuaria e que não importava se uma prática fosse capitalista ou socialista, se aumentasse a produtividade, essa prática deveria ser encorajada. Uma célebre frase de Deng ilustra o pragmatismo do governo chinês: "Não importa se o gato é preto ou branco, se o gato pega o rato, ele é um bom gato”. Segundo Xu Wu (2002, p. 13), Deng também "convocou o povo a deixar de lado o argumento ideológico e fazer do 'fazer dinheiro' a prioridade número um.” Esse foi o fim das relações públicas com características socialistas sob a liderança do partido. "Desde então, o desenvolvimento das relações públicas entrou em uma fase mais estável, com um movimento em direção à profissionalização" (NI CHEN, 1996, p. 127).

Segundo as pesquisas de campo realizadas por Ni Chen (1996), relações públicas, assim como outras influências ocidentais, difundiram-se primeiramente a partir das cidades costeiras do sul (província de Guangdong, cuja capital é Guangzhou - em português, Cantão); em seguida, para a capital comercial da China - Shanghai, e para a capital política - Beijing (Pequim). E só depois se expandiu para outras capitais mais tradicionais, como Nanjing. Esse dado é importante para empresas e organizações estrangeiras que estejam pensando em estratégias de alcance da China. Basicamente, a maioria das novidades que chegam à China segue esse itinerário: Shenzhen e Guangzhou (em Guangdong), Shanghai, Beijing e depois outras localidades.

Ni Chen (1996) adotou o modelo de Grunig (1994) das relações públicas excelentes, para traçar um perfil das práticas na China, e chegou a interessantes conclusões. Fatores como idade, gênero (masculino), educação e status dentro de uma organização estavam relacionados com a posição de gerenciamento e à comunicação assimétrica de duas mãos. Quanto mais alta a posição, mais ênfase se dava à função de gerenciamento da comunicação. E quanto mais velho, mais autoridade e responsabilidade o gerente acumulava, revelando uma característica cultural chinesa de valorização dos mais velhos em relação aos mais jovens. Ni Chen (1996) notou ainda que os profissionais de Relações Públicas no governo assumiam mais o papel de técnicos de comunicação e que os profissionais da área privada tinham mais facilidade para assumir funções de gerenciamento estratégico de comunicação.

Em comparação com o Ocidente, a prática de relações públicas na China é muito recente, e o guanxi é um dos grandes desafios à prática de Relações Públicas no estilo norte-americano naquele 
país. Todos os autores que tratam sobre aspectos da sociedade chinesa e sobre relações públicas na China, abordam invariavelmente essa questão. Para Yan (1996 apud HACKLEY; DONG, 2001, p. 16), guanxi pode ser definido como "uma rede de relacionamentos pessoais, estrategicamente construída."

Ni Chen (1996, p. 147) afirma que "guanxi é uma rede de relacionamentos pessoais que se tornou tão importante que as pessoas frequentemente não conseguem fazer nada sem conexões". Xu Wu (2002, p. 22) considera que um guanxi bem estabelecido pode garantir o sucesso da pessoa que se assenta no centro da rede. Ambos consideram que um bom guanxi pode ajudar uma empresa a conseguir uma cobertura positiva da mídia, adquirir políticas favoráveis dos burocratas oficiais; e mais importante, tal rede de relações pessoais entrelaçada como um guarda-chuva de proteção pode alertar para uma crise em potencial logo no começo, e eliminar qualquer consequência negativa no futuro. Quem tem mais poder hierarquicamente é responsável por proteger o mais fraco, que por sua vez retribuirá favores no futuro. É um sistema de trocas sociais que se distingue do sistema ocidental, pelo valor que os chineses conferem à importância de se pagar favores. Portanto, a familiaridade, a similaridade e a amizade são as bases para o desenvolvimento do guanxi chinês. Xu Wu (2002, p. 16) revela ainda que:

O guanxi pode existir não apenas no nível individual, mas pode também se espalhar entre duas empresas ou entre organizações com interesses diversos. [...] guanxi não é um curto período de reciprocidade, mas um processo de integração e identificação de longo prazo. O intercâmbio de benefício recíproco entre duas partes marca o início de um relacionamento de nível mais alto, ao invés de ser o fim de um contrato temporário de negócios.

Há controvérsias quanto à influência do guanxi na prática de relações públicas na China. Hekley e Dong (2001) destacam que esta rede chinesa é fechada a um círculo privado ou invisível de relações. "Em contraste com a ética de relações públicas americanas, o guanxi chinês, baseado em laços pessoais frequentemente secretos, pode ser visto como negociatas feitas debaixo da mesa, ou como suborno" (HEKLEY; DONG, 2001, p. 19). Nesse sentido, Ni Chen (1996) ressalta que realmente essa prática tão intrínseca à cultura chinesa parece "assimétrica à primeira vista, com sua ênfase no status e em relações pré-estabelecidas, mas ela incorpora princípios de simetria quando analisada em profundidade" (CULBERTSON; NI CHEN, 1996, p. 147).

As principais diferenças, apontadas por Hakley e Dong (2001), entre a prática das relações públicas americanas e o guanxi chinês podem ser analisadas em cinco dimensões: "1) privado x público; 2) fechado x aberto; 3 ) invisível $\mathrm{x}$ visível; 4) interpessoal $\mathrm{x}$ mídia de massa; 5) laços de amizade x centrado em princípios" (HAKLEY; DONG, 2001, p. 18). Enquanto os esforços das relações públicas americanas seriam no sentido da comunicação voltada para públicos, o guanxi se concentraria nas redes privadas de relacionamento. As relações públicas americanas buscam adotar uma política de portas abertas em sincronia com uma comunicação aberta, em contrataste com o ambiente fechado e secreto das práticas de guanxi. Um indivíduo chinês pode afirmar que tem guanxi, mas dificilmente pode mostrá-lo. Já as relações públicas americanas são visíveis, principalmente por meio da comunicação de massa.

Hakley e Dong (2001) enfatizam que o guanxi chinês é interpessoal e os canais de comunicação utilizados para o seu desenvolvimento seguem essa característica, enquanto os canais das relações públicas americanas são os meios de comunicação de massa.

É importante ressaltar a importância do guanxi sobre a questão do controle do governo chinês sobre a mídia do país. Tal controle limita a credibilidade da mídia (visivelmente parcial) e dificulta o acesso (à mídia) de muitas organizações como uma forma de comunicação com seus públicos. Assim, o guanxi acaba transformandose numa alternativa para o fortalecimento das relações de uma empresa com seus públicos.

Como se percebe, em decorrência desse aspecto histórico e cultural arraigado na sociedade chinesa, a grande dificuldade em relação à China é estabelecer um limite claro entre o que é público e o que é privado. O guanxi permeia as relações sociais e influencia diretamente todos os aspectos da esfera pública. Xu Wu (2002, p. 16) destaca que "é quase impossível requerer do povo chinês que diferencie claramente a parte pública e a parte privada de seu comportamento social." 
O público é, sem dúvida, um dos principais componentes do campo das relações públicas. $\mathrm{Na}$ China, o ambiente e os fatores culturais influenciam na definição de públicos. Xu Wu (2002, p. 15) ressalta que o fato da identidade de o indivíduo chinês estar na coletividade, não implica necessariamente a constituição de públicos - grupos bem informados, socialmente responsáveis e politicamente engajados. Afinal, nem espaço para isso há na atual organização política do país, em que o poder é centralizado nas mãos de um único partido e dissidentes são reprimidos pela força.

Assim, a cultura chinesa pode ser classificada como de "alto contexto". Ihator (2000, p. 39) explica que "toda comunicação acontece em um contexto social e em modos verbais e não-verbais. A cultura determina qual modo - verbal ou não verbal predomina.” Já para Hall (1976 apud IHATOR, 2000 , p. 79), "uma comunicação de alto contexto é aquela em que a maior parte da comunicação está ou no contexto físico ou internalizada na pessoa, enquanto que muito pouco está na parte codificada, explícita à mensagem.” Essa forma de comunicação diferencia-se bastante de culturas de baixo contexto, como a americana, em que a maior parte da informação está mesmo no código verbal explícito.

\section{O caso do instituto Brasil-China}

Fundado em 2003 na cidade de CuritibaPR, o Instituto Brasil-China de Intercâmbio Comercial, Tecnológico e Cultural foi uma associação civil sem fins lucrativos, que visou incentivar o intercâmbio sino-brasileiro, encerrando suas atividades em 2006.

Desde a sua fundação, o Instituto BrasilChina contou com uma diretoria de comunicação, voltada a posicionar o Instituto como um centro de referência nas relações entre os públicos brasileiros e chineses. Nesse sentido, os conhecimentos sobre a realidade sócio-cultural chinesa dentro da perspectiva das relações públicas internacionais e das práticas de relações públicas nesse país, foram decisivos para o sucesso das políticas e estratégias das ações comunicativas.

\section{Busca da simetria}

Em conformidade com a proposta de Grunig (1994) para as relações públicas excelentes, o equilíbrio entre os interesses dos públicos e os da organização foi um dos alicerces da comunicação do Instituto Brasil-China. Para que tal simetria ocorresse, foi necessário incluir em todas as estratégias e programas propostos canais de feedback que permitissem que os diversos públicos expressassem seus interesses e expectativas, críticas e sugestões. Assume-se o fato de que o equilíbrio completo de interesses nem sempre se faz totalmente na prática. Entretanto, esta busca de equilíbrio serviu como padrão para todas as ações de comunicação do Instituto Brasil-China.

\section{Conceito de cultural Awareness (ou consciência cultural)}

O Instituto Brasil-China se configurou como uma entidade que lidava com públicos biculturais, e possivelmente multiculturais. Isso afetou a comunicação de forma direta. A começar pelas barreiras impostas pelos diferentes idiomas. Dependendo do tipo de ação ou instrumento que pudesse vir a ser utilizado, seria necessário um cuidado especial com traduções e intérpretes. Havia que se prever o uso de português e mandarim, e muito possivelmente de inglês, em eventos, convites, cartazes, folders, página na Internet, etc.

Além disso, era preciso enxergar as nuances da cultura, do comportamento e das atitudes quando brasileiros e chineses estivessem interagindo mediante a intercessão do Instituto. Perceber as diferenças e rejeitar simplificações estereotipadas, quebrar falsos mitos e estar sintonizado nas mudanças pelas quais o Brasil, a China e o mundo passam dia após dia: todos esses fatores foram considerados quando no planejamento e execução de programas de comunicação.

\section{Fortalecimento do guanxi}

O guanxi é parte integrante da cultura chinesa, por isso foi fundamental investir nos relacionamentos existentes e iniciar outros. $\mathrm{O}$ instituto estabeleceu contatos e promoveu intensamente a aproximação entre Brasil e China por meio de palestras, feiras de negócios, exibições, projetos culturais e acadêmicos e reuniões sociais.

A instituição buscou ampliar parcerias com órgãos governamentais, tanto da China quanto do Brasil, para conquistar legitimidade perante seus públicos. Iniciou parcerias como o Governo do Estado do Paraná, com o Consulado da China no 
Brasil, localizado em São Paulo/SP, e com o IBECAP-Instituto Brasileiro de Estudos sobre a Ásia e Pacífico no Rio de Janeiro/RJ. A Câmara de Comércio e Indústria Brasil-China, Câmara BrasilChina de Desenvolvimento Econômico, Associação Cultural Chinesa do Paraná e a Câmara de Negócios Brasil-China do Paraná foram potenciais parceiros.

\section{CONSIDERAÇÕES FINAIS}

Nas últimas décadas, a globalização tornou-se realidade, como antecipou McLuhan na sua teoria sobre a "aldeia global". Essa nova configuração mundial promoveu o surgimento dos estudos em relações públicas internacionais, que embora avancem com relativa rapidez, ainda necessitam de mais pesquisas e aprofundamento.

De acordo com as pesquisas de Wakefield (1996), a cultura exerce grande influência nos trabalhos de relações públicas num contexto internacional, constituindo uma das principais linhas de pesquisa e ação. Para a realização de políticas e estratégias de comunicação para o Instituto BrasilChina, e para qualquer instituição que se relacione com públicos internacionais, é fundamental considerar preponderantemente os aspectos culturais, evitando estereótipos e generalizações, sobretudo ao se trabalhar com uma nação que possui uma riquíssima tradição cultural como a China.

Em chinês, a expressão que designa relações públicas é "Gonggong guanxi". Como se viu, o guanxi é uma rede de construção de relacionamentos privados, baseada nos princípios de Confúcio sobre as relações sociais. A noção de espaço público é um conceito ocidental. Aliandose a palavra "guanxi" a "gonggong", ou seja, público, pode-se concluir que a atividade de relações públicas está sempre tentando equilibrar interesses públicos e privados. Esse estudo das relações públicas em outros países, como no caso da China, acaba contribuindo para o conhecimento geral das relações públicas, ampliando as possibilidades de atuação dos profissionais da área.

Conhecer um pouco melhor um outro lado do mundo ensina um pouco mais sobre a própria humanidade e sobre nossa própria realidade como pesquisadores e profissionais. Percebemos que enxergar as semelhanças e as diferenças do outro é descobrir um pouco mais sobre nós mesmos.

\section{REFERÊNCIAS}

CASTELLS, M. Fim de milênio: a era da informação: economia, sociedade e cultura. São Paulo: Paz e Terra, 1999.

CULBERTSON, H. M; NI CHEN. International public relations: a comparative analisys. Mahwah: Lea Publishers, 1996.

CULTURE GRAMS. Concise, reliable up-to date country reports on 200 cultures of the world. Newcastle: Axion Press, 2003. Available in: <www.culturegrams.com>. Access in: 15 jun. 2008. (Não paginado).

GRUNIG, J. E. (Ed.). Excellence in public relations and communications management. Hillsdale: Lawrence Erlbaum Associates, 1994.

HACKLEY, C. A.; DONG, Q. American public relations networking encounters China's Guanxi. Public Relations Quarterly, Rhinebeck, v. 46, n. 2, p. 16-19, 2001.

HEATH, R. L. (Ed.). Handbook of public relations. Thousand Oaks: Sage Publications, 2001.

IHATOR, A. Understanding the cultural patterns of the world: an imperative in implementing strategic international PR programs. Public Relations Quarterly, Rhinebeck, v. 45, n. 4, p. 38-44, 2000.

JIANG, S. O Brasil visto da China. Veja, São Paulo, ano 40, n. 26, p. 11-15, 4 jul. 2007.

NI CHEN. Public relations in China: the introduction and development of na occupational field. In: CULBERTSON, H. M; NI CHEN. International public relations: a comparative analisys. Mahwah: Lea Publishers, 1996.

TAYLOR, M. International public relations: opportunities and challenges for the $21^{\text {st }}$. century. In: HEATH, R. L. (Ed.). Handbook of public relations. Thousand Oaks: Sage Publications, 2001. p. 625-637.

TU WEI MING. Múltiplas modernidades: uma investigação preliminar sobre as implicações da modernidade no leste da Ásia. In: HARRISON, L.; HUNTINGTON, S. A cultura importa. Rio de Janeiro; São Paulo: Record, 2002. p. 355-365. 
WAKEFIELD, R. I. Interdisciplinary theoretical foundations for international public relations. In: CULBERTSON, H. M.; NI CHEN. International public relations: a comparative analysis. Mahwah: Lea Publishers, 1996. p. 1-29.

WILCOX, D. et al. Public relations strategies and tactics. 7th ed. Boston: Allyn, 2003.

XU WU. D. PR in China: a 2001 version: concepts, practices and some misperceptions. Public Relations Quarterly, Rhinebeck, v. 47, n. 2, p.10-18, 2002.

Recebido: 13/04/2008

Received: 04/13/2008

Aprovado: $14 / 05 / 2008$

Approved: 05/14/2008 